\title{
PRODUÇÃO DE VÍDEO EDUCATIVO PARA PREVENÇÃO DE LINFEDEMA: RELATO DA EXPERIÊNCIA DE UM PROJETO DE EXTENSÃO
}

\section{AUDIOVISUAL RESOURCE PRODUCTION FOR HEALTHCARE: EXPERIENCES OF A UNIVERSITY OUTREACH PROJECT TO PREVENT LYMPHEDEMA}

\author{
Mariana Maia de Oliveira Sunemi* \\ ORCID: https://orcid.org/0000-0001-6910-9387 \\ Arnaldo Pinto Júnior** \\ ORCID: https://orcid.org/0000-0003-2516-9761 \\ Thaise da Paz Cardoso dos Santos*** \\ ORCID: https://orcid.org/0000-0002-3218-3556 \\ Luis Otávio Zanatta Sarian**** \\ ORCID: https://orcid.org/0000-0002-9554-6131 \\ Maria Teresa Pace do Amaral***** \\ ORCID: https://orcid.org/0000-0003-2563-5822
}

\section{Resumo}

Objetivou-se descrever e contextualizar as etapas do projeto de extensão Vídeo Educativo como Recurso para Prevenção de Linfedema Secundário ao Tratamento de Câncer Mamário. Este artigo se refere ao relato de experiência do projeto aprovado e financiado pela Pró-Reitoria de Extensão e Assuntos Comunitários (PREAC), da Unicamp, composto pelas etapas: constituição da equipe interprofissional; diálogos com mulheres submetidas ao tratamento oncológico; escolha da técnica e produção do vídeo; apresentação do material nas organizações não governamentais parceiras do projeto. A equipe executora - composta por sujeitos das áreas da saúde, educação e comunicação social - pautou-se em referenciais da educação interprofissional em saúde, a partir de práticas colaborativas. As experiências vivenciadas favoreceram o cuidado integral centrado no indivíduo; incentivaram a educação permanente como estratégia para a prática profissional em saúde; fortaleceram a articulação entre universidades e organizações não governamentais envolvidas; e promoveram o trabalho em equipe na perspectiva da prática colaborativa.

Palavras-chave: Linfedema; Prevenção de doenças; Recursos audiovisuais; Educação interprofissional; Práticas interdisciplinares.

\begin{abstract}
The aim of this study was to describe and contextualize the phases of the Educational Video outreach project as a Resource for the Prevention of Lymphedema Secondary to the Treatment of Breast Cancer. This manuscript is an experience report of the project approved and financed by Pro-Rectory of Extension and Community Affairs (PREAC), Unicamp, composed of the following steps: constitution of the interprofessional team; dialogues with women undergoing cancer treatment; choice of technique and video production; presentation of the material in the nongovernmental partner organizations. The executing team - including subjects from the areas of health, education and social communication - was based on references of interprofessional education in health and collaborative practices. The experiences favored comprehensive care centered on the individual; encouraged permanent education as a strategy for professional health practice; strengthened the link between the universities and non-governmental organizations involved; and promoted teamwork in the perspective of collaborative practice.
\end{abstract}

Keywords: Lymphedema; Disease prevention; Audiovisual aids; Interprofessional education; Interdisciplinary placement.

Data recebimento: 03/05/2021
* Professora da Universidade Federal de Minas Gerais (UFMG), Belo Horizonte - MG, Brasil. E-mail: marimfo@yahoo.com.br ** Professor da Universidade Estadual de Campinas (UNICAMP), Campinas - SP, Brasil. E-mail: apjfe@unicamp.br

*** Professora da Faculdade Maria Milza (FAMAM), Governador Mangabeira - Bahia, Brasil. E-mail: thai.dapaz@hotmail.com **** Professor da Universidade Estadual de Campinas (UNICAMP), Campinas - SP, Brasil. E-mail: sarian@gmail.com ***** Professora da Universidade Federal de São Paulo (UNIFESP), Santos - SP, Brasil. E-mail: mtp.amaral@unifesp.br 


\section{Introdução}

De acordo com Instituto Nacional de Câncer (INCA), o câncer de mama é o mais frequente e comum tumor maligno entre as mulheres, excluindo-se os tumores de pele não melanoma. Para o Brasil, no biênio 2020-2021, estima-se 66.280 casos novos por ano, com um risco de 56,33 casos a cada 100 mil mulheres (BRASIL, 2020).

O Ministério da Saúde, por meio de programas e políticas públicas a serem executadas pelo Sistema Único de Saúde (SUS), vem buscando priorizar a prevenção e o diagnóstico precoce, assim como facilitar cada vez mais o acesso das mulheres ao tratamento oncológico e seu acompanhamento. Como parte deste plano, a Política Nacional de Atenção Básica define a organização de Rede de Atenção à Saúde como estratégia para um cuidado integral e direcionada às necessidades de saúde de determinada população. Além disso, prioriza também a responsabilização na atenção contínua e integral pelo cuidado multiprofissional (BRASIL, 2013).

Com o objetivo de organizar o fluxo dos indivíduos de acordo com suas necessidades, a atenção deve ser realizada através das linhas de cuidado. Com relação ao câncer de mama, a organização da linha de cuidado envolve intervenções na promoção da saúde, na prevenção, no tratamento, na reabilitação e nos cuidados paliativos, englobando os diferentes níveis de atenção à saúde, com o objetivo de alcançar bons resultados clínicos baseados em evidências, a custos compatíveis (BRASIL, 2013).

As possibilidades terapêuticas propostas para as mulheres diagnosticadas com câncer de mama, tais como abordagem axilar e radioterapia, podem ser também precursoras de complicações, como o linfedema. Estas complicações comprometem a funcionalidade e qualidade de vida, merecendo, portanto, um olhar mais atento da equipe de saúde, no sentido de prevenção, diagnóstico precoce e encaminhamento adequado para o tratamento.

O linfedema é considerado condição crônica (HAVENS et al., 2021) associada à disfunções físico-funcionais - inchaço, sensação de peso, dor (KORUCU et al., 2020) - e psicológicas - angústia, ansiedade, alteração da imagem corporal e impacto social (ZHAO et al., 2021) - interferindo significativamente na qualidade de vida das mulheres submetidas ao tratamento do câncer de mama (INTERNATIONAL SOCIETY OF LYMPHOLOGY, 2020; TEMUR; KAPUCU, 2019; ZHAO et al., 2021). Ocorre por acúmulo de fluido rico em proteína no espaço intersticial e pode ser causado por dissecção de linfonodos axilares, biópsia de linfonodo sentinela, radioterapia, infecção, obesidade (INTERNATIONAL SOCIETY OF LYMPHOLOGY, 2020), além de formação de seroma no pós-operatório, quimioterapia no membro homolateral à cirurgia ou estágio avançado da doença (RIBEIRO PEREIRA et al., 2017). Em estudo observacional com 964 mulheres, os autores verificaram incidência de 13,5\% em dois anos de seguimento, 30,2\% em cinco anos e 41,1\% em dez anos (RIBEIRO PEREIRA et al., 2017). 
Além dos fatores de risco já mencionados, a ausência de informações compreensíveis sobre o linfedema, a não realização de exercícios e a não adesão ao autocuidado para preveni-lo, podem aumentar o risco de aparecimento desta complicação (DAVIES et al., 2020; RIBEIRO PEREIRA et al., 2017; TEMUR; KAPUCU, 2019). Em estudo prospectivo com 141 mulheres com diagnóstico de linfedema, os autores observaram que apenas 31\% das participantes tiveram adesão maior que $75 \%$ às orientações de autocuidado em um período de 12 meses (BROWN et al., 2014). Outros autores observaram que as mulheres que receberam informações para tais práticas relataram menos sintomas e mais ações para medidas de redução de risco através de modificações no estilo de vida, do que aquelas que não o fizeram (LU et al., 2015).

Quando instalado, o tratamento padrão ouro para o linfedema é a Terapia Física Complexa (INTERNATIONAL SOCIETY OF LYMPHOLOGY, 2020), abordagem contínua e cara, realizada por fisioterapeutas para reduzir o edema e consistência do membro. Embora esta complicação não tenha cura, seu controle é possível (LIANG et al., 2020; TEMUR; KAPUCU, 2019).

Sendo assim, promover a adesão às práticas preventivas e favorecer o reconhecimento dos sinais e sintomas iniciais é fundamental para evitar a instalação desta complicação (BELMONTE et al., 2018; TEMUR; KAPUCU, 2019). Considerando a diversidade sociocultural das mulheres, os profissionais da saúde devem refletir sobre a utilização de diferentes linguagens, com vista a ampliar a divulgação de informações e orientações que contribuam efetivamente para o conhecimento, a atitude e a prática de cuidado para prevenção de linfedema. Diante da diversidade mencionada, múltiplas formas de leitura, apropriação e significação das informações divulgadas são construídas. Assim, ainda não há consenso na literatura em relação aos melhores métodos de comunicação a respeito da prevenção de linfedema.

As informações transmitidas verbalmente ou por meio de material escrito não têm contribuído significativamente para a mudança de comportamento das mulheres, necessária à adesão ao autocuidado (LU et al., 2015; TEMUR; KAPUCU, 2019). Já a utilização de um produto com as especificidades da linguagem audiovisual, elaborado para sensibilizar o públicoalvo, pode proporcionar maior clareza e compreensão das temáticas identificadas como necessidades de cuidado (DALMOLIN et al., 2016), contribuindo para a desejada mudança de comportamento frente à complicação. Diante disso, considerou-se a produção de um recurso audiovisual que pudesse contribuir para o conhecimento sobre os sinais e sintomas iniciais do linfedema, e estimular o protagonismo e a autonomia da mulher no processo de cuidado em saúde, com consequente melhora da qualidade de vida. A produção deste vídeo dialogou com referenciais da educação interprofissional em saúde e trabalho em equipe, a partir das práticas colaborativas. 
Este relato de experiência tem por objetivo descrever e contextualizar as etapas do projeto de extensão Vídeo Educativo como Recurso para Prevenção de Linfedema Secundário ao Tratamento de Câncer Mamário.

\section{Relato da experiência}

\section{Justificativa do projeto de extensão e etapas da construção do vídeo educativo}

A existência de conhecimento limitado sobre linfedema e seus fatores de risco por parte das mulheres submetidas ao tratamento oncológico mamário, e a dificuldade de acesso a um cuidado de qualidade (BUKI et al., 2021), podem interferir na adesão às práticas preventivas desta complicação. Além disso, barreiras pessoais, como a falta de tempo e de disposição, foram identificadas como entraves para a participação de mulheres em grupos educativos sobre o câncer de mama e seu tratamento (LOYOLA et al., 2017).

Partindo de tais evidências, como também da necessidade clínica e rotineira de fisioterapeutas envolvidos com a prática oncológica mamária, é que se pensou em produzir uma ferramenta - vídeo educativo - que pudesse implementar a atenção à saúde de mulheres submetidas ao tratamento oncológico mamário.

Assim, docentes e pesquisadores da Universidade Estadual de Campinas (Unicamp) e Universidade Federal de São Paulo (Unifesp) - Campus Baixada Santista - elaboraram um projeto de extensão intitulado Vídeo Educativo como Recurso para Prevenção de Linfedema Secundário ao Tratamento de Câncer Mamário. O projeto foi submetido ao edital de número 10 da Pró-Reitoria de Extensão e Assuntos Comunitários (PREAC) da Unicamp, tendo sido aprovado e financiado pela referida Pró-Reitoria. Seu desenvolvimento ocorreu entre os meses de setembro de 2016 e agosto de 2017.

O grupo de trabalho constituído para a execução do projeto foi composto por três fisioterapeutas, sendo uma docente, uma profissional e uma pós-graduanda; dois médicos docentes; um docente da área da Educação; e cinco estudantes da área de Comunicação Social - um mestrando e quatro graduandas do curso de Midialogia. Três organizações não governamentais (ONGs) participaram do projeto, a saber: Projeto Renascer, em São José do Rio Pardo; Grupo Rosa e Amor, em Valinhos e Instituto Neo Mama de Combate ao Câncer de Mama, em Santos. extensão.

O Quadro 1 reúne o conjunto das atividades desenvolvidas no decorrer do projeto de 
Quadro 1 - Atividades desenvolvidas

\begin{tabular}{|c|c|c|c|}
\hline Etapas de execução do projeto & Objetivos & Estratégias & Desdobramentos \\
\hline $\begin{array}{l}\text { Etapa 1: A constituiç̧ão da } \\
\text { equipe interprofissional }\end{array}$ & $\begin{array}{l}\text { Estimular a troca de } \\
\text { ideias e saberes entre } \\
\text { os diferentes profis- } \\
\text { sionais e acadêmicos } \\
\text { acerca dos temas: } \\
\text { câncer de mama / } \\
\text { linfedema / vídeo } \\
\text { educativo }\end{array}$ & Rodas de conversa & $\begin{array}{l}\text { Levantamento das } \\
\text { potencialidades e } \\
\text { desafios da produção } \\
\text { do vídeo }\end{array}$ \\
\hline $\begin{array}{l}\text { Etapa 2: diálogo com mulheres } \\
\text { assistidas em serviço público } \\
\text { especializado em assistência } \\
\text { oncológica }\end{array}$ & $\begin{array}{l}\text { Conhecer o que as } \\
\text { mulheres sabem sobre } \\
\text { o linfedema e suas } \\
\text { perspectivas em } \\
\text { relação ao tratamento } \\
\text { oncológico }\end{array}$ & $\begin{array}{l}\text { Entrevistas semi } \\
\text { dirigidas }\end{array}$ & $\begin{array}{l}\text { Elaboração do roteiro } \\
\text { do vídeo }\end{array}$ \\
\hline $\begin{array}{l}\text { Etapa 3: escolha da técnica e } \\
\text { produção do vídeo }\end{array}$ & $\begin{array}{l}\text { Definir a arte áudio- } \\
\text { visual a partir do } \\
\text { custo/benefício e } \\
\text { objetivo do projeto; } \\
\text { produzir o vídeo }\end{array}$ & $\begin{array}{l}\text { Apresentação, por } \\
\text { acadêmicos do curso } \\
\text { de Midialogia, da } \\
\text { técnica de desenho } \\
\text { com giz e lousa; } \\
\text { escrita do texto a ser } \\
\text { narrado; gravação e } \\
\text { edição do material }\end{array}$ & $\begin{array}{l}\text { Produção da } 1^{\text {a }} \text { versão } \\
\text { do vídeo; avaliação do } \\
\text { vídeo pela equipe; } \\
\text { aprovação da versão } \\
\text { final }\end{array}$ \\
\hline $\begin{array}{l}\text { Etapa 4: Apresentação do } \\
\text { vídeo nas ONGs parceiras do } \\
\text { projeto }\end{array}$ & $\begin{array}{l}\text { Apresentar o vídeo e } \\
\text { discutir sobre as } \\
\text { potencialidades deste } \\
\text { recurso como } \\
\text { estratégia para } \\
\text { orientação das práticas } \\
\text { preventivas de } \\
\text { linfedema }\end{array}$ & $\begin{array}{l}\text { Roda de conversa com } \\
\text { os profissionais } \\
\text { integrantes das } \\
\text { instituições parceiras }\end{array}$ & $\begin{array}{l}\text { Publicação do vídeo } \\
\text { na plataforma } \\
\text { Youtube }\end{array}$ \\
\hline
\end{tabular}

Fonte: Autores 


\section{Análise das etapas de execução do projeto}

\section{Etapa 1 - A constituição da equipe interprofissional}

O primeiro movimento importante a ser destacado nesta etapa é a transformação que ocorreu em relação ao envolvimento dos integrantes com o projeto, ou seja, a transformação de grupo de trabalho em equipe interprofissional. A literatura aponta que um grupo se caracteriza por um conjunto de pessoas reunidas num mesmo espaço e tempo, com um objetivo em comum, e cada indivíduo mantendo entre si vínculos e relações afetivas de diversas naturezas (CIAMPONE; PEDUZZI, 2000).

Já uma equipe vai além da coesão grupal entre seus membros, visa a eficácia, a eficiência e a efetividade da assistência e dos cuidados em saúde produzidos pelos profissionais, permitindo que as ações sejam desenvolvidas em complementaridade (PEDUZZI et al., 2016; PEDUZZI; LEONELLO; CIAMPONE, 2016).

Nesse contexto, e diante de um cenário em que os sistemas de saúde estão fragmentados e com dificuldades para atender as necessidades de saúde, a Educação Interprofissional (EIP) vem sendo incentivada como possibilidade para melhorar a qualidade e o resultado dos atendimentos em saúde. Assim, trabalhou-se, neste projeto, com a perspectiva da EIP para a formação em saúde, que "ocorre quando estudantes de duas ou mais profissões aprendem sobre os outros, com os outros e entre si para possibilitar a colaboração eficaz e melhorar os resultados na saúde" (OMS, 2010, p. 7).

Um dos pilares da EIP é a prática colaborativa, que acontece quando os "profissionais de diferentes áreas prestam serviços com base na integralidade da saúde, envolvendo os pacientes e suas famílias, cuidadores e comunidades para atenção à saúde da mais alta qualidade em todos os níveis da rede de serviços" (OMS, 2010, p. 13).

Houve a necessidade, então, de maior aproximação entre os participantes do projeto, a fim de se conhecer e se inteirar a respeito das diferentes profissões e respectivas atuações no cenário e contexto em que as ações seriam realizadas. Nesse sentido, conceitos sobre competências específicas, comuns e colaborativas foram discutidos.

Barr (1998), um dos primeiros pesquisadores a discutir a educação interprofissional a partir do modelo de competências, conceitua competências específicas como aquelas que compreendem o conhecimento que distingue uma determinada profissão e que complementa 
as outras. As competências comuns podem ser desenvolvidas por todas as profissões da saúde. E as competências colaborativas são capazes de promover um espaço coletivo de atuação no qual acontece a efetiva colaboração entre os diferentes profissionais envolvidos no trabalho, instituições, usuários, familiares e comunidades. Desse modo, compreender as diferentes competências que compõem a EIP permitiu aos integrantes da equipe melhor articulação das ideias acerca da produção do vídeo educativo para prevenção de linfedema.

Reconhecendo os pressupostos da EIP, a equipe desenvolveu discussões em quatro encontros, cuja temática norteadora foi o câncer de mama, linfedema e vídeo educativo. A estratégia utilizada nesta etapa inicial foi a roda de conversa, que possibilita encontros dialógicos em que os participantes se expressam, escutam os outros e a si mesmos, e desenvolvem a autonomia por meio do compartilhamento de informações e de reflexões para a ação (ADAMY et al., 2018).

Questões técnicas e de custo em relação à produção de vídeo também foram apresentadas e discutidas nestes encontros. Assim, a equipe dimensionou os desafios e as potencialidades da elaboração de uma ferramenta capaz de orientar pacientes com diferentes perspectivas de leitura e interpretação da linguagem audiovisual.

\section{Etapa 2 - Diálogo com mulheres submetidas ao tratamento oncológico}

Para que o objetivo do vídeo fosse cumprido - tornar-se uma ferramenta que pudesse contribuir para a adesão das mulheres às práticas preventivas de linfedema -, o material a ser produzido precisaria estar em consonância com algumas demandas de mulheres com câncer de mama. Desse modo, a equipe entendeu ser fundamental ouvir pacientes para conhecer os diferentes contextos socioculturais, familiares e de conhecimento acerca do linfedema, valorizando, assim, a prática com atenção centrada no paciente (ACP).

A ACP constitui um dos domínios essenciais para a colaboração interprofissional e elemento primordial do trabalho em equipe. Além disso, na perspectiva ampliada do cuidado, contribui para a atenção à saúde de forma holística, integral e orientada a partir das necessidades em saúde (AGRELI; PEDUZZI; SILVA, 2016).

Foram convidadas para o diálogo cinco mulheres de diferentes faixas etárias e em distintas fases do tratamento oncológico - pós-operatório precoce, em tratamento quimioterápico ou em seguimento clínico -, acompanhadas na Seção de Fisioterapia do hospital Prof. Dr. José Aristodemo Pinotti - Centro de Atenção Integral à Saúde da Mulher (Caism). O interlocutor da equipe que conduziu os encontros foi o estudante de pós-graduação em Midialogia que, em um espaço reservado, recebeu cada mulher separadamente e procurou levantar informações específicas sobre o quadro clínico geral; sentimentos frente ao diagnóstico e tratamento do 
câncer; expectativas de retorno às atividades do cotidiano; perspectivas do tratamento; e conhecimento sobre as possíveis complicações do tratamento, principalmente em relação ao linfedema.

A análise e discussão das narrativas obtidas foram trazidas para uma nova rodada de reuniões da equipe. O cotidiano das mulheres em processo de tratamento oncológico trouxe à luz reflexões em relação ao cuidado oferecido pelos serviços de saúde, às perspectivas futuras desta população - seus medos, angústias, comprometimentos físicos, psicológicos e sociais - e em relação à qualidade de vida durante e após o tratamento oncológico. Isto contribuiu para maior sensibilização dos estudantes e profissionais das áreas de Educação e Comunicação Social a respeito do processo de enfrentamento do câncer de mama e compreensão da complexidade dos casos oncológicos em questão, ressignificando, assim, seus papéis na elaboração do recurso audiovisual e na assistência à saúde.

Dialogar sobre as diferentes percepções dos integrantes da equipe, principalmente os que não são da área da saúde, certamente favoreceu a construção de um roteiro adequado e factível. A equipe se organizou para a etapa de construção do roteiro a partir de discussões em relação às potencialidades e dificuldades da produção pretendida. Neste momento, mais uma vez ficou clara a potencialidade do trabalho em equipe interprofissional, através das práticas colaborativas e com a atenção centrada no paciente.

$\mathrm{O}$ roteiro contemplou questões relacionadas à epidemiologia; tratamento do câncer de mama; linfedema - conceito, fatores de risco, sinais e sintomas iniciais; e orientações de autocuidado para sua prevenção. Com o intuito de complementar este conteúdo, a equipe optou por incluir no roteiro trechos de entrevistas com outras mulheres com e sem linfedema e em diferentes contextos de trabalho e/ou atividades de vida diária, a fim de aproximar o conteúdo do vídeo à realidade das espectadoras.

\section{Etapa 3 - Escolha da técnica e produção do vídeo}

No processo de definição da técnica explorada na comunicação visual, os estudantes de Midialogia sugeriram a utilização de giz e lousa para a produção do vídeo. Essa sugestão baseou-se no custo final e na característica lúdica das imagens, abordando o conteúdo a ser transmitido. A animação em 2D desenvolvida na sequência de quadros e os efeitos do comtraste entre o fundo escuro da lousa e as cores dos gizes utilizados contemplaram as expectativas dos integrantes da equipe, principalmente no que refere à forma clara e atrativa das representações gráficas, em conjunto com o áudio explicativo (Figura 1). 
Mariana Maia de Oliveira Sunemi et al.

Figura 1 - Sequência de imagens que faz referência à necessidade de realizar exercícios com os membros superiores (A); e ao fator de risco de infecção, indicando que há a necessidade de cuidar da pele (B).
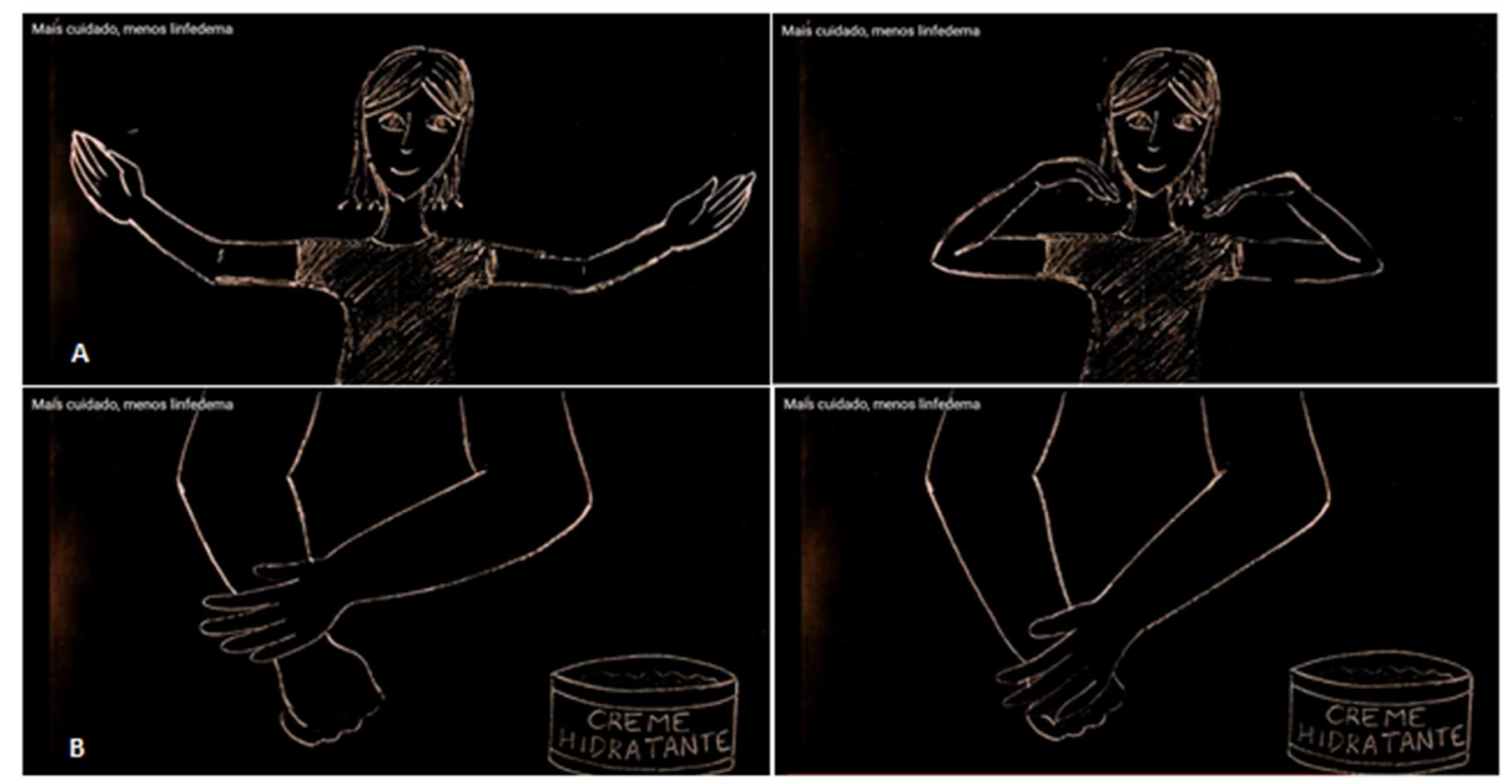

Fonte: Autores

Feita a escolha da técnica, e a partir do roteiro elaborado, a equipe entendeu que o conteúdo deveria ser apresentado em, aproximadamente, 20 minutos, tempo considerado adequado para transmitir as informações sobre linfedema e práticas preventivas. Além disso, o tempo estipulado e o formato escolhido - composto tanto pela perspectiva lúdica dos desenhos quanto da exibição intercalada de trechos de entrevistas - tiveram como objetivo manter a atenção das espectadoras, evitando a dispersão e favorecendo a compreensão do conteúdo exibido.

Os integrantes da equipe pertencentes à área de Midialogia iniciaram as filmagens a partir da sequência de desenhos produzidos com giz e lousa, enquanto os integrantes das áreas da saúde e educação elaboraram o texto a ser narrado, a partir do roteiro produzido.

Finalizadas estas ações, foi feita a gravação da narração do texto e, na sequência, realizou-se a edição do material até aqui produzido. Sendo assim, uma primeira versão do vídeo, composta por animação, texto narrado referente ao conteúdo e trechos de entrevistas mesclados ao longo do vídeo, foi produzida e apresentada à equipe. Após a discussão e análise criteriosa do material pela equipe, pequenos ajustes foram realizados, sendo feita a aprovação final do vídeo educativo. 


\section{Etapa 4 - Apresentação do vídeo educativo para as instituições parceiras do projeto}

As três instituições parceiras são organizações não governamentais que assistem mulheres submetidas ao tratamento oncológico mamário, e que possuem vínculo com as universidades que integram o projeto. Em suas dinâmicas de assistência, contam com o apoio de fisioterapeutas, psicólogos, assistentes sociais e nutricionistas.

Nesse contexto, as fisioterapeutas integrantes da equipe propuseram às instituições parceiras a realização de uma oficina de aproximadamente 3 horas, com os seguintes objetivos: (a) apresentar o vídeo e sensibilizar os profissionais envolvidos com o cuidado oncológico em relação à importância das práticas preventivas para o linfedema; (b) refletir e discutir sobre como conduzir a orientação dessas práticas, principalmente evitando o caráter proibitivo de algumas ações realizadas pela paciente; (c) esclarecer possíveis dúvidas dos profissionais participantes em relação à prática preventiva; (d) fomentar a discussão sobre possíveis questionamentos por parte das mulheres assistidas e contribuir para a reflexão em conjunto de adequação dos esclarecimentos.

Durante as oficinas, foram trazidas à discussão pela equipe do projeto as potencialidades de se apresentar o vídeo educativo para as mulheres, em grupo, independentemente do período de tratamento em que se encontram - pós-operatório precoce, tardio, ou ainda, durante a quimioterapia ou radioterapia. De acordo com o Cadernos Humaniza SUS (2010), do Ministério da Saúde, o grupo não é simplesmente agrupar pessoas numa sala, mas, sim, um componente importante da organização das práticas e do projeto terapêutico das pessoas. Além disso, os grupos facilitam a comunicação do profissional com o usuário, estreitando essa relação. E isso se deve ao fato de que o espaço de grupo propicia que o saber esteja nas pessoas e não centrado em um profissional de saúde, mas também nele.

Com relação à doença, o cuidado em grupo pode trazer bons resultados para o manejo clínico de determinada condição. Os usuários podem sentir maior abertura para expor e dividir com os demais integrantes a experiência que têm no manejo da doença, trazendo dúvidas e curiosidades que somente o compartilhar poderia propiciar (BRASIL, 2010).

\section{Reflexões acerca do vídeo educativo como estratégia de educação em saúde}

Um dos maiores desafios - e também potencialidade - deste projeto foi o envolvimento de acadêmicos e profissionais das áreas da saúde, educação e comunicação. Os profícuos diálogos e as tensões geradas entre seus integrantes ao longo do processo de planejamento e produção do vídeo proporcionaram experiências significativas para todos, sobretudo quando 
foi compreendido e incorporado pela equipe o objetivo comum da integralidade do cuidado às mulheres submetidas ao tratamento oncológico mamário.

A EIP em saúde vem sendo discutida em instituições de ensino superior e viabilizada a partir de diferentes estratégias que compõem os projetos políticos pedagógicos, projetos de extensão, Programa de Educação pelo Trabalho (PET), entre outras ações. Tais estratégias têm como prioridade o trabalho em equipe - pressuposto essencial para a integralidade do cuidado - através das práticas colaborativas. Essa integralidade pode ser compreendida como parte central da interdisciplinaridade e interprofissionalidade, e tem sido facilitada por meio da troca de saberes e práticas comuns (AZEVEDO; PEZZATO; MENDES, 2017). Dialogar com outros saberes que não o da saúde, neste projeto, contribuiu ainda mais para uma atenção voltada à integralidade do cuidado e centrada no indivíduo, preceitos basilares do SUS.

Pensando na prevenção do linfedema secundário ao tratamento oncológico mamário, as orientações quanto aos cuidados com o membro operado, a realização de exercícios e o reconhecimento de sinais e sintomas característicos dessa complicação seriam suficientes para diminuir sua incidência. Entretanto, na prática clínica observa-se a dificuldade de adesão, por parte das mulheres, às orientações recebidas.

Basicamente, as estratégias de prevenção têm sido em torno de palestras educativas acerca do linfedema, panfletos e manuais com ilustrações e orientações escritas, além de informações passadas verbalmente em consultas ambulatoriais (TEMUR; KAPUCU, 2019). Muitas vezes, essas estratégias não levam em consideração o contexto social, econômico e cultural vivido por essas mulheres. Mediante tais aspectos, percebe-se que os recursos e as estratégias utilizados por profissionais de saúde para a prevenção do linfedema ainda não são suficientes para produzir a mudança de comportamento necessária para a apreensão das informações pela mulher e, consequentemente, a incorporação e prática dessas informações em suas atividades cotidianas.

As estratégias audiovisuais - como os vídeos educativos em saúde - desenvolvem atitudes perceptivas e facilitam a construção do conhecimento por se tratar de um material com recursos visuais e auditivos que estimulam a imaginação e servem como mediador do aprendizado. Além disso, os vídeos podem transformar a informação à medida que despertam a curiosidade em públicos de diferentes contextos sociais, econômicos e culturais (MORTOLA et al., 2021).

No contexto da educação em saúde, Rosa et al. (2019) inferem que o recurso audiovisual contribui para a promoção em saúde, educação e aprendizado para quem o assiste, proporcionando mudanças no cotidiano de cuidado. Além disso, favorece a participação do sujeito no processo educativo, contribuindo para sua autonomia. Assim, e considerando as demandas das mulheres por melhor qualidade de vida após o diagnóstico e tratamento do 
câncer de mama, o vídeo educativo busca sensibilizar o público-alvo com conhecimentos necessários, em linguagem acessível.

Segundo pesquisadores que analisaram estudos da área de educação (AGRA et al., 2019), a aprendizagem significativa é um processo pelo qual novas informações se articulam à estrutura de conhecimento específica e relevante de um indivíduo. Nesse sentido, para se alcançar tal processo com um determinado público, a nova informação apresentada deve ser inteligível, condição que potencializa o estabelecimento de conexões com conhecimentos relevantes previamente construídos. Assim, quando ocorre a interação entre a nova informação e uma estrutura de conhecimento específica, pode-se trabalhar com o conceito de aprendizagem significativa, independente da área de atuação. Em se tratando deste projeto, a partir da exposição de informações claras e acessíveis sobre o linfedema, buscou-se estimular as pacientes a produzirem novas compreensões do referido quadro clínico, com vistas às mudanças nas posturas e comportamentos frente à condição.

Portanto, para que as mulheres percebam a importância de colocar em prática as ações de prevenção, faz-se necessário superar a barreira do conhecimento e do vocabulário científico que, invariavelmente, separam pacientes e profissionais de saúde. Cada indivíduo tem sua história, formação escolar e/ou acadêmica perpassadas por contextos familiares e socioculturais diversos. Por isso, neste projeto, trabalhou-se com o desenvolvimento de estratégias que pudessem contribuir para a apropriação significativa das orientações, gerando, assim, mudanças de comportamento da mulher tratada por câncer de mama.

Os recursos audiovisuais com especificidades educativas, produzidos com o objetivo de informar e orientar pacientes oncológicos, favorecem a ampliação do conhecimento e o desenvolvimento das práticas de autocuidado; da compreensão e da tomada de decisão dos pacientes, reduzindo o estresse e melhorando o bem-estar psicológico (NATHOO, 2017).

Além disso, os profisssionais das instituições envolvidas no diálogo com a equipe executora do projeto manifestaram que a reflexão sobre suas práticas foi fundamental para alcançar melhores resultados no cuidado à saúde. Essa atitude reflexiva não apenas favoreceu maior integração entre as diferentes especialidades, como também fortaleceu a perspectiva interprofissional e interdisciplinar nos ambientes de trabalho.

A produção deste vídeo educativo compõe a primeira parte de um projeto maior, que tem como objetivo principal verificar o conhecimento, a atitude e a prática de mulheres em relação à prevenção de linfedema secundário ao câncer de mama. A etapa seguinte prevê a realização de um ensaio clínico com a utilização deste vídeo. 


\section{Considerações Finais}

As experiências vivenciadas durante o processo de produção do vídeo educativo e discussão com as instituições parceiras proporcionaram avanços na reflexão acerca de estratégias para o cuidado e atenção às mulheres submetidas ao tratamento oncológico mamário. Ao trabalharmos com princípios da EIP, procuramos reunir os conhecimentos comuns e específicos de diferentes áreas - saúde, educação e midialogia - para compor um produto com linguagem audiovisual adequada, acessível e capaz de sensibilizar as mulheres assistidas.

Vale destacar ainda o quão potente foi este projeto no sentido de ampliar o diálogo entre os profissionais de saúde e as pacientes; favorecer o cuidado integral centrado no indivíduo; incentivar a educação permanente como estratégia para uma prática profissional com melhores resultados na atenção à saúde; aproximar universidades e ONGs envolvidas; e articular o trabalho de estudantes de diferentes áreas a um cenário de prática que possibilitou o exercício de competências na perspectiva da atuação interprofissional colaborativa.

Atualmente, o vídeo encontra-se disponível para acesso público na plataforma YouTube (Mais cuidado, menos linfedema - YouTube).

\section{Referências}

ADAMY, E. K. et al. Validação na teoria fundamentada nos dados: rodas de conversa como estratégia metodológica. Revista Brasileira de Enfermagem, Brasília, v. 71, n. 6, p. 3121 3126, 2018. DOI: https://doi.org/10.1590/0034-7167-2017-0488. Disponível em: https://www.scielo.br/j/reben/a/5ZfVsKjNX6znX3rZPgvWmTz/?lang=en. Acesso em: 11 jan. 2021.

AGRA, G. et al. Análise do conceito de Aprendizagem Significativa à luz da Teoria de Ausubel. Revista Brasileira de Enfermagem, Brasília, v. 72, n. 1, p. 258-265, 2019. DOI: https://doi.org/10.1590/0034-7167-2017-0691. Disponível em: https://www.scielo.br/j/reben/a/GDNMjLJgvzSJKtWd9fdDs3t/?lang=en. Acesso em: 16 abr. 2021.

AGRELI, H. F.; PEDUZZI, M.; SILVA, M. C. Atenção centrada no paciente na prática interprofissional colaborativa. Interface - Comunicação, Saúde e Educação, Botucatu, v. 20, n. 59, p. 905-916, out./dez. 2016. DOI: http://dx.doi.org/10.1590/1807-57622015.0511. Disponível em: https://www.scielo.br/j/icse/a/sXhwQWKsZGzrQqT4tDryCXC/?lang=pt. Acesso em: 21 jan. 2021. 
AZEVEDO, A. B.; PEZZATO, L. M.; MENDES, R. Formação interdisciplinar em saúde e práticas coletivas. Saúde em Debate, Rio de Janeiro, v. 41, n. 113, p. 647-657, abr./jun. 2017. DOI: https://doi.org/10.1590/0103-1104201711323. Disponível em: https://www.scielo.br/j/sdeb/a/qBXChrFm9MGGDSjD69nk5mC/?lang=pt. Acesso em: 10 fev. 2021.

BARR, H. Competent to collaborate: Towards a competency-based model for interprofessional education. Journal of Interprofessional Care, v. 12, n. 2, p. 181-187, 1998. DOI: https://doi.org/10.3109/13561829809014104. Disponível em: https://www.tandfonline.com/doi/abs/10.3109/13561829809014104. Acesso em: 3 jun. 2021.

BELMONTE, R. et al. Prospective study of shoulder strength, shoulder range of motion, and lymphedema in breast cancer patients from pre-surgery to 5 years after ALND or SLNB. Support Care Cancer, v.26, n. 9, p. 3277-3287, set. 2018. DOI: https://doi.org/10.1007/s00520-018-4186-1. Disponível em: https://link.springer.com/ article/10.1007/s00520-018-4186-1. Acesso em: 9 abr. 2021.

BRASIL. Ministério da Saúde. Estimativa 2020: Incidência de câncer no Brasil. [Internet]. Instituto Nacional de Câncer José Alencar Gomes da Silva (INCA). 2020. Disponível em: https://www.inca.gov.br/sites/ufu.sti.inca.local/files/media/document/estimativa-2020incidencia-de-cancer-no-brasil.pdf

BRASIL. Ministério da Saúde. Secretaria de Atenção à Saúde. Departamento de Atenção Básica. Controle dos cânceres do colo do útero e da mama, Secretaria de Atenção à Saúde, Departamento de Atenção Básica. 2. ed. Brasília: Ministério da Saúde, 2013.

BRASIL. Ministério da Saúde. Secretaria de Atenção à Saúde. Política Nacional de Humanização. 256 p.: il. - (Série B. Textos Básicos de Saúde) (Cadernos HumanizaSUS; v. 2). Brasília: Ministério da Saúde, 2010.

BROWN, J. C. et al. Prescription and adherence to lymphedema self-care modalities among women with breast cancer-related lymphedema. Support Care in Cancer, v. 22, n. 1, p. 135-143, 2014. DOI: https://doi.org/10.1007/s00520-013-1962-9. Disponível em: https://link.springer.com/article/10.1007/s00520-013-1962-9. Acesso em: 22 mar. 2021. 
BUKI, L. P. et al. "I never heard anything about it": Knowledge and psychosocial needs of Latina breast cancer survivors with lymphedema. Womens Health (London), v. 17, p. 1-11, jan./dez. 2021. DOI: https://doi.org/10.1177/17455065211002488. Disponível em: https://journals.sagepub.com/doi/10.1177/17455065211002488. Acesso em: 2 abr. 2021.

CIAMPONE, M. H. T.; PEDUZZI, M. Trabalho em equipe e trabalho em grupo no Programa de Saúde da Família. Revista Brasileira de Enfermagem, Brasília, v. 53, n. especial, p. 143-147, dez. 2000. DOI: https://doi.org/10.1590/S0034-71672000000700024. Disponível em: http://reben.com.br/revista/artigos/?volume=53\&numero=7. Acesso em: 15 maio 2021.

DALMOLIN, A. et al. Vídeo educativo como recurso para educação em saúde a pessoas com colostomia e familiares. Revista Gaúcha de Enfermagem, Porto Alegre, v. 37, p. 1-9, 2016. Disponível em: https://seer.ufrgs.br/RevistaGauchadeEnfermagem/article/view/68373/40824 . Acesso em: 9 mar. 2021.

DAVIES, C. et al. Interventions for Breast Cancer-Related Lymphedema: Clinical Practice Guideline From the Academy of Oncologic Physical Therapy of APTA. Physical Therapy, v. 100, n. 7, p. 1163-1179, jul. 2020. DOI: http://dx.doi.org/10.1093/ptj/pzaa087. Disponível em: https://pubmed.ncbi.nlm.nih.gov/32589208/. Acesso em: 20 jul. 2021.

Executive Committee International Society of Lymphology. The diagnosis and treatment of peripheral lymphedema: 2020 consensus document of the International Society of Lymphology. Lymphology, v. 53, n. 1, p. 3-19, 2020. Disponível em:

https://journals.uair.arizona.edu/index.php/lymph/article/view/23775. Acesso em: 2 fev. 2021.

HAVENS, L. M. et al. Use of technology to facilitate a prospective surveillance program for breast cancer-related lymphedema at the Massachusetts General Hospital. Mhealth. V. 7, p. 1-14, jan. 2021. DOI: http://dx.doi.org/10.21037/mhealth-19-218. Disponível em: https://mhealth.amegroups.com/article/view/44504/html. Acesso em: 20 jul. 2021. 
KORUCU, T. S. et al. Comparison of Shoulder-Arm Complex Pain, Function, and Scapular Dyskinesia in Women With and Without Unilateral Lymphedema After Breast Cancer Surgery. Clin Breast Cancer. v. 21, n. 3, e285-e293, out. 2020. DOI:

http://doi.org/10.1016/j.clbc.2020.10.008. Disponível em: https://www.clinical-breastcancer.com/article/S1526-8209(20)30267-6/fulltext. Acesso em: 21 jul. 2021.

LIANG, M. D. et al. Manual lymphatic drainage for lymphedema in patients after breast cancer surgery: A systematic review and meta-analysis of randomized controlled trials. Medicine, v. 99, n. 49, e23192, dez, 2020. DOI: https://doi.org/10.1097/md.0000000000023192.

Disponível em: https://journals.lww.com/md-journal/Fulltext/2020/12040/

Manual lymphatic drainage for lymphedema in.30.aspx. Acesso em: 13 maio 2021.

LOYOLA, E. A. C. et al. Grupo de reabilitação: benefícios e barreiras sob a ótica de mulheres com câncer de mama. Texto \& Contexto Enfermagem, Florianópolis, v. 26, n. 1, p. 1-10, 2017. DOI: http://dx.doi.org/10.1590/0104-07072017003250015. Disponível em: https://www.scielo.br/j/tce/a/mv5Xq65DtDPtyz67ZQVhsKd/?lang=en. Acesso em: 26 mar. 2021.

LU, S. R. et al. Role of physiotherapy and patient education in lymphedema control following breast cancer surgery. Therapeutics and Clinical Risk Management, v. 11, p. 319-327, fev. 2015. DOI: http://doi.org/10.2147/TCRM.S77669. Disponível em: https://www.dovepress.com/role-of-physiotherapy-and-patient-education-in-lymphedemacontrol-foll-peer-reviewed-fulltext-article-TCRM. Acesso em: 2 jan. 2021.

MORTOLA, L. A. et al. Vídeo educativo sobre a quimioterapia oncológica: tecnologia na educação em saúde. Ciência, Cuidado e Saúde, v. 20, p. 1-7, 2021. Disponível em: https://periodicos.uem.br/ojs/index.php/CiencCuidSaude/issue/view/1879. Acesso em: 3 abr. 2021.

NATHOO, D. Video material as an effective educational tool to address informational and educational needs of cancer patients undergoing radiation therapy. Journal of Cancer Education, v. 32, n. 2, p. 219-227, 2017. DOI: http://doi.org/10.1007/s13187-015-0933-x. Disponível em: https://link.springer.com/article/10.1007\%2Fs13187-015-0933-x. Acesso em: 20 dez. 2020.

ORGANIZAÇÃO MUNDIAL DA SAÚDE. Marco para ação em educação interprofissional e prática colaborativa. 2010. Disponível em: https://www.who.int/hrh/nursing midwifery/en /. Acesso em: 29 abr. 2020.

PEDUZZI, M. et al. Trabalho em equipe, prática e educação interprofissional. Clínica médica. $2^{\mathrm{a}}$ ed. Barueri: Manole, v. 1, p. 1-9, 2016. 
PEDUZZI, M.; LEONELLO, V. M.; CIAMPONE, M. H. T. Trabalho em equipe e prática colaborativa. Gerenciamento em enfermagem. Rio de Janeiro: Guanabara Koogan, p. 10313, 2016.

RIBEIRO PEREIRA A. C. P.; KOIFMAN, R. J.; BERGMANN, A. Incidence and risk factors of lymphedema after breast cancer treatment: 10 years of follow-up. The Breast, v. 36, p. 67-73, dez. 2017. DOI: http://doi.org/10.1016/j.breast.2017.09.006. Disponível em: https://www.thebreastonline.com/article/S0960-9776(17)30578-7/fulltext. Acesso em: 2 jan. 2021.

ROSA, B. V. C. et al. Desenvolvimento e validação de tecnologia educativa audiovisual para famílias e pessoas com colostomia por câncer. Texto \& Contexto Enfermagem, Florianópolis, v. 28, p. 1-15 2019. DOI: https://doi.org/10.1590/1980-265X-TCE-2018-0053. Disponível em: https://www.scielo.br/j/tce/i/2019.v28/. Acesso em: 27 nov. 2020.

TEMUR, K.; KAPUCU, S. The effectiveness of lymphedema self-management in the prevention of breast cancer-related lymphedema and quality of life: A randomized controlled trial. European Journal of Oncology Nursing, v. 40, p. 22-35, jun. 2019. DOI: http://doi.org/10.1016/j.ejon.2019.02.006. Disponível em:

https://www.ejoncologynursing.com/article/S1462-3889(19)30021-3/fulltext. Acesso em: 12 nov 2020.

ZHAO, H. et al. Breast cancer-related lymphedema patient and healthcare professional experiences in lymphedema self-management: a qualitative study. Supportive Care in Cancer, jul, 2021.DOI: http://doi.org/10.1007/s00520-021-06390-8. Disponível em: https://link.springer.com/article/10.1007\%2Fs00520-021-06390-8. Acesso em: 22 jul. 2021. 